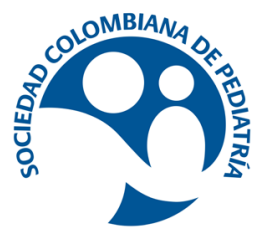

\title{
Original
}

\section{Necesidades percibidas en el paciente pediátrico hospitalizado}

\section{Francy Cantor Cruz ${ }^{a}$, Hannie Jhuliana Niño Vargas ${ }^{b}$ María Ligia Cifuentes Álvarez ${ }^{c}$ Sandra Patricia Zorro Cerónd ${ }^{d}$ Nancy Yomayusa González ${ }^{e}$}

a. Psicóloga Msc(c) Epidemiología clínica. Centro Iberoamericano de Investigación en Salutogénesis y Bienestar. Instituto Global de Excelencia Clínica (IGEC). Keralty;

b. Licenciada en Educación Especial, Msc Educación. Clinica Colsanitas, Keralty;

c. Licenciada en Psicopedagogía. Clinica Colsanitas, Keralty;

d. Psicóloga, Especialista en Psicología de la Salud. Clinica Colsanitas, Keralty;

e. MD. Esp. en Medicina interna y nefrología, Grupo de Medicina Traslacional. Instituto Global de Excelencia Clínica. Clínica Colsanitas, Keralty.

INFORMACIÓN DEL ARTÍCULO

Historia del artículo:

Recibido el 13 de marzo de 2020

Aceptado el 07 de noviembre 2020

Palabras clave:

Hospitalización

atención centrada en el paciente

evaluación de necesidades

experiencias negativas en la infancia..

\section{R E S U M E N}

Introducción: La hospitalización es una situación que los niños pueden experimentar como negativa, intimidante o adversa, contribuyendo a la aparición de necesidades que pueden pasar desapercibidas durante la atención médica y que en estancias hospitalarias largas son más evidentes. Objetivo: Describir la experiencia en la identificación y abordaje de las necesidades percibidas en niños hospitalizados, utilizando una estrategia basada en la lúdica durante el año 2018 en tres clínicas de Bogotá. Métodos: Se realizó un estudio de métodos mixtos, con análisis de datos retrospectivo sobre los regi psicopedagógica basada en la lúdica. Resultados: Los pacientes pediátricos hospitalizados independientemente de su edad o patología de ingreso, presentan durante las tres primeras semanas de hospitalización necesidades relacionadas con el estado de ánimo y la interacción con el cuidador. Los cuidadores también presentan necesidades relacionadas con el manejo de la incertidumbre por el diagnóstico y el pronóstico del niño, la interrupción en la dinámica y funcionamiento familiar, y preocupaciones por la atención médica y por aspectos psicosociales y educativos de los niños durante su estancia en el hospital Conclusiones: Las estrategias pedagógicas basadas en la lúdica son un recurso valioso con el que las instituciones prestadoras de servicios podrían mejorar la experiencia de los niños que requieren ser hospitalizados y de sus cuidadores.

\footnotetext{
*Autor para correspondencia. Francy Cantor Cruz Correo electrónico: ftcantor@keralty.com
}

Como Citar: Cantor Cruz F, Niño Vargas HJ, Cifuentes Álvarez ML, Zorro Cerón SP, Yomayusa González N. Necesidades percibidas en el paciente pediátrico hospitalizado . Pediatr. 2020;53(2):64-71. 


\section{Perceived necessities of pediatric hospitalized patients}

\section{A B S T R A C T}

Keywords:

Hospitalization

patient-centered care

needs assessment

adverse childhood experiences
Introduction: Hospitalization is a situation that children can experience as negative, intimidating, or adverse, contributing to the appearance of needs that may go unnoticed during medical care and are more evident in extended hospital stays. Objective: To describe the experience in identifying and addressing hospitalized children's perceived needs, using a play-based strategy during 2018, in three clinics in Bogotá. Methods: A mixed-methods study was carried out, with retrospective data analysis on the care medical records carried out in 2018. The selection of patients was consecutive and convenient. For identifying the children's necessities, structured interviews were carried out, and a psycho-pedagogical strategy based on the play was implemented. Results: Pediatric hospitalized patients, regardless of their age or admission pathology, present during the first three weeks of hospitalization needs related to mood and interaction with the caregiver. Caregivers also present needs related to the management of uncertainty due to the child's diagnosis and prognosis, the interruption in family dynamics and functioning, and concerns about medical care, psychosocial and educational aspects of the children during their stay in the hospital Conclusions: Educational strategies based on play are a valuable resource with which service-providing institutions could improve the experience of children requiring hospitalization and their caregivers.

\section{Introducción}

La hospitalización para los pacientes pediátricos es una situación en la que viven un período de aislamiento de su entorno familiar y social, alejamiento de las personas significativas como cuidadores, hermanos, amigos, compañeros de clase e interrupción repentina y abrupta de sus rutinas y actividades cotidianas. El nuevo ambiente en el que el niño se ve inmerso unido al malestar fisiológico de la enfermedad, pueden contribuir a la vivencia de la hospitalización como algo negativo, intimidante o adverso (1, 2), llegando a producir alteraciones del sueño y del apetito, aumento del estrés, alteraciones emocionales como ansiedad o depresión, conductas de agresividad y oposición, problemas de atención y rechazo a la medicación, así como el desarrollo de terrores y temores a la interacción con el personal sanitario $(3,4,5)$.

Aunque este tipo de atención puede ser infrecuente en la vida de los niños, una proporción importante de ellos anualmente lo requieren. Para el 2017, el Centro Nacional de Estadísticas de Salud de Estados Unidos reportó una frecuencia de hospitalización del $1.9 \%$ de los niños menores de 18 años atendidos en ese año (6), en Colombia, algunos reportes de la asociación colombiana de empresas de medicina integral muestran tasas de hospitalización en sus pacientes de 20 por cada 100000 niños menores de 1 año y de 1.7 por cada 100000 niños de 5 a 14 años, siendo los menores de 5 años quienes generan una demanda más alta del servicio (7).

La vivencia de la hospitalización como algo adverso y la falta de acciones para mitigar su impacto, pueden contribuir a la aparición de necesidades en los niños, las cuales pueden pasar desapercibidas durante la atención clínica, pero hacerse más evidentes a largo plazo, especialmente en los casos que por la condición clínica requieren hospitalizaciones frecuentes o prolongadas afectando la calidad de vida de los niños $(8,9)$ la de sus cuidadores (10).

Uno de los primeros reportes que se tienen sobre este impacto, es el síndrome de "hospitalismo" presentado por los niños durante la hospitalización, para el cual estrategias desde la psicopedagogía hospitalaria surgieron como respuesta en Europa para 1940; unas primeras adaptaciones de estas estrategias se realizaron en Colombia por el Hospital de la Misericordia (HOMI), implementando la intervención por pedagogos en el pabellón de niños quemados (4). Algunas de las herramientas de intervención utilizadas desde este enfoque son las aulas hospitalarias para el fortalecimiento de los procesos educativos interrumpidos por la hospitalización, la risoterapia para el mejoramiento del estado de ánimo, y la intervención basada en la lúdica para el fortalecimiento de diversos aspectos psico-socioafectivos $(11,12)$.

En este contexto, la identificación y abordaje de las necesidades en los pacientes a través de enfoques novedosos como el que proporciona la psicopedagogía hospitalaria podrían brindar oportunidades de mejora para los servicios de hospitalización pediátrica que les permita acercarse cada vez más a una atención centrada en el paciente (13) y que a su vez impacten positivamente en la experiencia y el transcurrir de la hospitalización para el niño y sus cuidadores. Por esto, nuestro objetivo fue describir la experiencia en la identificación y abordaje de las necesidades percibidas en niños hospitalizados menores de 18 años utilizando una estrategia 
basada en la lúdica, durante el año 2018 en tres clínicas de Bogotá.

\section{Métodos}

Estudio descriptivo de métodos mixtos, el cual permite dar mayor profundidad al análisis de los datos, favoreciendo la interpretación de los resultados al combinar las perspectivas y técnicas de investigación cuantitativa y cualitativa, para comprender mejor el fenómeno de interés investigado (14).

\section{Población}

Pacientes menores de 18 años, hospitalizados por cualquier causa entre los periodos de febrero a mayo o entre agosto de noviembre del año 2018 en alguna de las tres clínicas incluidas en este estudio, situadas en Bogotá D.C.

\section{Selección de los pacientes}

Los pacientes fueron seleccionados de manera consecutiva y a conveniencia en los servicios de hospitalización y de urgencias pediátricas de las clínicas. A través de un primer contacto con los cuidadores, se explicó sobre el programa de psicopedagogía hospitalaria y la disponibilidad en la institución para realizar la intervención basada en la lúdica durante su permanencia en las instalaciones si era de interés para el niño y la familia.

\section{Criterios de inclusión y exclusión}

Se incluyeron todos aquellos niños y cuidadores en los cuales se obtuvo el consentimiento verbal para el desarrollo de la estrategia lúdica. Se excluyeron a los pacientes que al momento de desarrollar la actividad lúdica estuvieran dormidos, sedados o con baja disposición para realizar las actividades debido a alguna condición física.

\section{Descripción de la estrategia basada en la lúdica}

Se realizó una revisión de literatura para identificar las propuestas de intervención existentes basadas en la lúdica y su impacto en la hospitalización. La revisión de la literatura fue evaluada, por dos expertos en psicopedagogía y psicología, quienes cuentan con 20 años de experiencia en atención a niños en contextos sanitarios. Basados en los resultados de la revisión, la experticia profesional de los evaluadores, las dimensiones del desarrollo infantil postuladas por Vygotsky (15) y Piaget (16), las alteraciones psicosociales, emocionales y comportamentales descritas en la literatura y los lineamientos de primera infancia del desarrollo integral del niño del Ministerio de Educación de Colombia (17) se construyó la entrevista estructurada para la identificación de necesidades y se determinaron las actividades de abordaje a realizar según los resultados y el rol del cuidador durante la intervención.
La estrategia hizo parte del Programa de Psicopedagogía Hospitalaria, abierto desde el 2001, como respuesta a las inquietudes del personal de salud y padres de familia frente al aburrimiento de los niños hospitalizados. Inicialmente se implementó en la Clínica Colsanitas Reina Sofia para pacientes de medicina prepagada y posteriormente se fue ampliando la atención a otros regímenes de aseguramiento y cobertura a otras clínicas con servicios de hospitalización pediátrica.

\section{Componentes de la estrategia}

La estrategia está conformada por seis etapas que se describen a continuación.

Etapa 1. Identificación de las necesidades: entrevista estructurada dirigida a los cuidadores, indagando por la condición clínica, características sociodemográficas del niño y las expectativas del cuidador frente al desarrollo de una estrategia lúdica en hospitalización (Anexo 1). De manera paralela, a través de observación participante se evaluó según la edad, el desarrollo de las habilidades psicomotoras, lingüísticas, cognitivas, el estado de ánimo del niño y la interacción niño-cuidador. A partir de la entrevista y la observación, el profesional determinó las necesidades existentes en los niños.

Etapa 2. Selección de la actividad de juego: La selección se realizó de acuerdo al tipo de necesidades identificadas. Así, en los casos en los que se identificó un desempeño menor al esperado para la edad del niño en una habilidad del desarrollo específica, la actividad de juego se enfocó específicamente a su reforzamiento. En los casos contrarios, en donde se identificó un desarrollo acorde a lo esperado para la edad del niño en todas las habilidades, la selección del juego fue guiada por las expectativas y/o preocupaciones del cuidador y se enfocó en potenciar o estimular las habilidades en general del niño. En los casos en los que el cuidador no tuviera una expectativa específica la selección del juego se enfocó en la estimulación de habilidades sociales, cognitivas y motrices.

Etapa 3. Desarrollo de la actividad de juego: La actividad de juego se desarrolló en las habitaciones del hospital en las que se encontraban los pacientes o en las salas de juego de las clínicas. Se utilizaron juegos comerciales o construidos por los profesionales en formación (Ver anexo 2). La actividad fue guiada y acompañada por los profesionales en formación vinculados a las clínicas como parte de sus prácticas profesionales en pedagogía infantil.

Etapa 4. Trabajo con cuidadores: Durante la evaluación y desarrollo de cada una de las actividades los cuidadores estuvieron presentes y en situaciones específicas como: percibir resistencia o bajo involucramiento de los niños en la actividad de juego, una interacción niño-cuidador pobre o interés del cuidador en participar de la actividad, fueron involucrados de manera activa por el profesional en formación. 
Etapa 5. Finalización: Al finalizar la actividad del juego, se retroalimentó al cuidador sobre la actividad lúdica realizada y se diligenciaron los resultados en la ficha técnica.

\section{Aplicación de la estrategia}

Todas las etapas se realizaron el mismo día con cada paciente, las cuales tuvieron una duración mínima de 1 hora y máxima de 2 horas 30 minutos. La variación en el tiempo estuvo relacionada con el involucramiento, la participación y disfrute del paciente y sus familiares en la actividad lúdica. Toda la estrategia fue desarrollada por profesionales de pedagogía infantil en formación, supervisados por los investigadores de este estudio.

\section{Análisis de la información}

Se realizó un análisis estadístico utilizando medidas de tendencia central y dispersión para las variables continuas según su distribución, evaluada con la prueba de ShapiroWilks y análisis de frecuencias con valores absolutos para las variables categóricas, para describir las características clínicas y sociodemográficas de los pacientes atendidos, los resultados obtenidos en la evaluación de las necesidades y los resultados del abordaje realizado. El software utilizado en este análisis fue R 3.1.5.

Para la información cualitativa, se realizó un análisis temático de la información contenida en los registros de la entrevista y se presentó a través de síntesis narrativa y frecuencias cuando fue posible hacerlo, utilizando el programa de Microsoft Excel®. La información de este estudio fue tomada de los registros disponibles en los programas de psicopedagogía hospitalaria de las clínicas y analizada de manera retrospectiva; los casos en los que el registro tuvo pérdidas en más del $20 \%$ de la información, se intentó recuperar consultando la historia clínica y otros archivos disponibles en el programa. Se excluyeron los registros en los que no se logró recuperar la información faltante.

\section{Consideraciones éticas}

Análisis retrospectivo de la información disponible en los registros previamente recolectados, por lo que no se requirió de consentimiento informado.

\section{Resultados}

\section{Caracterización de la muestra}

Un total de 472 niños hospitalizados fueron evaluados, de los cuales 32 no realizaron la actividad lúdica por indisposición del niño. Las causas fueron, dolor o estar dormido en el $53 \%$ $(n=17)$, por encontrarse en traslado (cirugía, radiología, tener orden de salida o cambio de habitación) en el $25 \%(n=8)$, por encontrarse en otra terapia en el $6.2 \%(n=2)$ y otras razones en el $15.6 \%(n=5)$.

Un total de 440 niños y sus cuidadores participaron de la estrategia lúdica completa, los menores tenían entre los 2 meses y 192 meses (16 años) de edad, siendo el $51.6 \%$ ( $n=227)$ de sexo femenino. La estancia hospitalaria tuvo un promedio de 3.4 días (DE 4.6) con un rango de 0 a 47 días.

Las patologías por las que estaban hospitalizados los niños fueron enfermedades respiratorias o del pulmón en el $50.2 \%$ $(n=221)$, enfermedades gastrointestinales en el $10.2 \%(n=45)$, enfermedades de la piel en el $6.8 \%(n=30)$, enfermedades infecciosas en el $6.36 \%(n=28)$, intervenciones quirúrgicas en el $5 \%(n=22)$, enfermedades de vías urinarias en el $5 \%(n=22)$, enfermedades del sistema nervioso en el $4.3 \%(n=19)$, enfermedades vasculares en el $3.4 \%(n=15)$, enfermedades óseas en el $2.3 \%(n=10)$, enfermedades psiquiátricas en el 1.6 $\%(n=7)$, enfermedades endocrinas en el $1 \%(n=6)$, enfermedades oncológicas en el $0.4 \%(n=2)$, otros diagnósticos en el $1 \%(n=4)$, que incluyen mordedura, desnutrición, intoxicación exógena, riesgo pérdida implante y en el $2 \%(n=9)$ no se obtuvo el diagnóstico de base.

Los cuidadores de los niños durante el desarrollo de la estrategia lúdica fueron, solo la madre en el $51.9 \%$ ( $n=241)$, madre y padre en el $14.9 \%(n=69)$, otros familiares en el $11.2 \%$ $(n=52)$, siendo abuelos $(n=28)$, padrastro $(n=3)$, niñera $(n=1)$, primos $(n=2)$, hermanos $(n=3)$, tíos $(n=15)$. Solo padre en el 9.3 $\%(n=43)$, madre y otro familiar en el $7.11 \%(n=33)$, abuelos del niño $(n=22)$, tíos del niño $(n=6)$, hermanos del niño $(n=3)$, amigo $(n=1)$, prima $(n=1)$ y el padre y otro familiar, siendo este la abuela en el $0.4 \%(n=2)$. En cuanto a la red de apoyo se encontró que las madres tuvieron apoyo de familiares en el $12 \%$ de los casos mientras que los padres lo tuvieron en un $4 \%$.

\section{Resultados de la identificación de las necesidades:}

En los pacientes evaluados y que participaron de toda la estrategia lúdica, se percibieron necesidades en el $14.5 \%$ relacionadas con el estado de ánimo tales como: decaimiento, aburrimiento, tristeza, irritación. Llanto incontrolable en el 8.4 $\%(n=37)$, necesidades relacionadas con la interacción entre paciente y cuidador en el $5.4 \%(n=24)$, necesidades de lenguaje o comunicación en el $1.1 \%(n=5)$ y cognitivas en el $0.7 \%(n=3)$. En el $85.4 \%$ ( $n=376)$ de los niños, no se percibieron necesidades y en ningún niño se percibieron necesidades psicomotoras.

\section{Análisis de las necesidades según la edad del niño}

Uno de cada diez niños hospitalizados independientemente de la etapa de desarrollo en la que se encontraba (grupo etario) tuvo una necesidad relacionada con el estado de ánimo. En uno de cada 20 niños hospitalizados entre 2 meses y 7 años se observaron necesidades en la interacción con su cuidador, así como en uno de cada 30 niños entre 8 a 11 años y en uno de cada 15 niños entre los 12 y 16 años. A continuación, se 
describen todas las necesidades percibidas agrupadas por edad según las etapas del desarrollo de Piaget. (Ver tabla 1).

\section{Análisis de las necesidades según el tiempo de hospitalización}

Desde el día cero, es decir el mismo día de ingreso hasta el día 20 o tercera semana de hospitalización, las necesidades más frecuentes están relacionadas con el estado de ánimo y alteración en la interacción con el cuidador. A partir de la cuarta semana hasta la séptima no se percibieron necesidades en los niños hospitalizados. En cuanto a las edades, entre la primera y la segunda semana se atendieron pacientes niños y adolescentes. A partir de la tercera semana variaron los grupos etarios, siendo solo niños o solamente adolescentes. (Ver tabla 2).

\section{Análisis de las necesidades según el diagnóstico clínico de} hospitalización

Las necesidades fueron percibidas en mayor proporción en el grupo de enfermedades gastrointestinales ( $28 \%$ ), seguidas por las enfermedades del sistema nervioso (26,31 \%), las enfermedades infecciosas (21,42\%), las intervenciones quirúrgicas (18,18\%), enfermedades respiratorias (10\%) y endocrinas (16,6\%). En seis grupos de enfermedades no se percibieron necesidades. (Ver tabla 3).

Análisis de las necesidades según el género

Las necesidades relacionadas con el estado de ánimo fueron mayores en los niños en comparación con las niñas $8.9 \%$
( $\mathrm{n}=19)$, en lenguaje y/o comunicación $0.9 \%(\mathrm{n}=2)$ y cognitivas $0.9 \%(n=2)$ teniendo una diferencia proporcional de $1 \%, 0.5 \%$ y $0.4 \%$ respectivamente. En las niñas las necesidades fueron mayores en interacción social 5.7 \% $(n=13)$ en comparación de $5.2 \%(n=11)$ con los niños.

\section{Análisis de las preocupaciones manifestadas por el cuidador}

El $44.8 \%(n=197)$ de los cuidadores manifestaron tener preocupaciones adicionales a las médicas. El $20.8 \%(n=41)$ estuvo relacionadas con el estado emocional del niño (desánimo, irritabilidad, intranquilidad del niño, llanto incontrolable, decaimiento, inquietud, extrañar el hogar, familiares o mascotas, estrés). El $15.7 \%(n=31)$ con los servicios médicos, tales como contagio de otra enfermedad, falta de diagnóstico preciso, inconformidad con el servicio, demora en la atención, incomodidad, el tiempo de hospitalización, alimentación y aseo de las instalaciones.

El $13 \%(n=26)$ por las actividades escolares, el $12.7 \%(n=25)$ por el desarrollo del niño (dificultad en la atención, lectura, lenguaje, escritura, motricidad gruesa y fina), el $10.6 \%(n=21)$ por la falta de actividades de entretenimiento o aburrimiento; en el $9.9 \%(n=20)$ por el comportamiento del niño (hiperactividad, falta de seguimiento de instrucciones o desobediencia, comportamientos desafiantes, dificultad en la convivencia y relacionamiento con otros niños, agresividad, timidez, falta de seguimiento de normas en el hogar). El $8.6 \%$ $(n=17)$ por la salud en general (recuperación, pérdida de otras terapias, cirugías o procedimientos programados previamente), el $4 \%(n=8)$ por el trabajo del cuidador, en el $3.5 \%(n=7)$ por la

\begin{tabular}{ccccccccc}
$\begin{array}{c}\text { Tabla 1. Descripción de las necesidades categorizadas según las etapas del desarrollo de Piaget. } \\
\begin{array}{c}\text { Edad } \\
\text { Dimensión }\end{array}\end{array}$ & $n$ & Psicomotor & Lenguaje \%(n) & $\begin{array}{c}\text { Estado de Ánimo } \\
\%(n)\end{array}$ & Cognitivo \%(n) & Interacción \%(n) & Ninguna $(n)$ \\
\hline 2 meses a 2 años & 108 & - & $1.8 \%(2)$ & $8.3 \%(9)$ & $1.8 \%(2)$ & $5.5 \%(6)$ & 89 \\
\hline 3 a 7 años & 193 & - & $1 \%(2)$ & $8.2 \%(16)$ & - & $5.7 \%(11)$ & 165 \\
\hline 8 a 11 años & 87 & - & - & $8.4 \%(7)$ & $1.1 \%(1)$ & $3.44 \%(3)$ & 78 \\
\hline 12 a 16 años & 52 & - & - & $9.6 \%(5)$ & - & $7.7 \%(4)$ & 45 \\
\hline
\end{tabular}

Tabla 2. Descripción de las necesidades categorizadas según el tiempo de hospitalización.

\begin{tabular}{|c|c|c|c|c|c|c|c|c|}
\hline $\begin{array}{l}\text { Días de hospitalización } \\
\text { (semanas) }\end{array}$ & N & Psicomotor & Lenguaje & $\begin{array}{c}\text { Estado de Animo } \\
\%(n)\end{array}$ & $\begin{array}{c}\text { Cognitiva } \\
\%(n)\end{array}$ & $\begin{array}{c}\text { Interacción } \\
\%(n)\end{array}$ & $\begin{array}{l}\text { Ninguna } \\
\text { (n) }\end{array}$ & $\begin{array}{l}\text { Rango edad } \\
\text { (años) }\end{array}$ \\
\hline 0 & 31 & - & - & $22.5 \%(7)$ & $9.7 \%$ (1) & $16.1 \%(5)$ & 19 & $1-11$ \\
\hline $1-6$ (1) & 336 & - & $0.9 \%$ (3) & $8 \%(27)$ & $0.6 \%(2)$ & $3.9 \%(13)$ & 292 & $0-16$ \\
\hline $7-13(2)$ & 40 & - & $2.5 \%(1)$ & $5 \%(2)$ & - & $10 \%(4)$ & 35 & $1-16$ \\
\hline $14-20(3)$ & 4 & - & - & $25 \%$ (1) & - & $25 \%(1)$ & 3 & $12-14$ \\
\hline $21-27(4)$ & 3 & - & - & - & - & - & 3 & $1-7$ \\
\hline $28-34(5)$ & 1 & - & - & - & - & - & 1 & 2 \\
\hline $35-41(6)$ & 0 & - & - & - & - & - & 0 & - \\
\hline $42-47(7)$ & 2 & - & - & - & - & - & 2 & $8-13$ \\
\hline
\end{tabular}




\begin{tabular}{|c|c|c|c|c|c|c|c|c|c|c|}
\hline Diagnóstico & N & $P M$ & $\begin{array}{l}\text { Lenguaje } \\
\%(n)\end{array}$ & $\begin{array}{c}\text { Estado de } \\
\text { Animo } \\
\%(n)\end{array}$ & $\begin{array}{l}\text { Cognitivo } \\
\%(n)\end{array}$ & $\begin{array}{c}\text { Interacción } \\
\%(n)\end{array}$ & $\begin{array}{l}\text { Total } \\
\%(n)\end{array}$ & $\begin{array}{l}\text { Ninguno } \\
(n)\end{array}$ & $\begin{array}{c}\text { Rango } \\
\text { hospitalización } \\
\text { (días) }\end{array}$ & $\begin{array}{l}\text { Rango } \\
\text { edad } \\
\text { (años) }\end{array}$ \\
\hline $\begin{array}{l}\text { Enfermedades } \\
\text { respiratorias }\end{array}$ & 221 & - & $1.3 \%(3)$ & $5.9 \%(13)$ & - & 3. $6 \%$ (8) & $10 \%(24)$ & 198 & $0-29$ & $0-15$ \\
\hline $\begin{array}{l}\text { Enfermedades } \\
\text { gastrointestinales }\end{array}$ & 45 & - & - & $15 \%(7)$ & $2.2 \%(1)$ & $11.1 \%(5)$ & $28 \%(13)$ & 33 & $0-16$ & $2-16$ \\
\hline $\begin{array}{l}\text { Enfermedades } \\
\text { infecciosas }\end{array}$ & 28 & - & - & $17.8 \%(5)$ & - & $3.6 \%$ (1) & $21.4 \%(6)$ & 23 & $0-10$ & $1-15$ \\
\hline $\begin{array}{l}\text { Intervención } \\
\text { quirúrgica }\end{array}$ & 22 & - & - & $9.1 \%(2)$ & - & $9.1 \%$ (2) & $18.2 \%$ (4) & 18 & $1-12$ & $3-15$ \\
\hline $\begin{array}{l}\text { Enfermedades del } \\
\text { sistema nervioso }\end{array}$ & 19 & - & - & $10.5 \%(2)$ & $10.5 \%(2)$ & $5.3 \%(1)$ & $26.3 \%$ (5) & 15 & $0-5$ & $1-15$ \\
\hline $\begin{array}{l}\text { Enfermedades } \\
\text { Endocrinas }\end{array}$ & 6 & - & - & $16.6 \%(1)$ & - & - & $16.6 \%(1)$ & 5 & $0-3$ & $2-10$ \\
\hline \multicolumn{11}{|c|}{ Enfermedades en las que no se percibieron necesidades } \\
\hline $\begin{array}{l}\text { Enfermedades de } \\
\text { la Piel }\end{array}$ & 24 & - & - & - & - & - & & 24 & $1-20$ & $1-13$ \\
\hline $\begin{array}{l}\text { Enfermedades de } \\
\text { vías urinarias }\end{array}$ & 20 & - & - & - & - & - & & 20 & $1-45$ & $0-12$ \\
\hline $\begin{array}{l}\text { Enfermedades } \\
\text { vasculares }\end{array}$ & 12 & - & - & - & - & - & & 12 & $1-14$ & $2-15$ \\
\hline $\begin{array}{l}\text { Enfermedades } \\
\text { óseas }\end{array}$ & 8 & - & - & - & - & - & & 8 & $0-47$ & $5-13$ \\
\hline $\begin{array}{l}\text { Enfermedades } \\
\text { psiquiátricas }\end{array}$ & 7 & - & - & - & - & - & & 7 & $1-2$ & $11-15$ \\
\hline $\begin{array}{l}\text { Enfermedades } \\
\text { Oncológicas }\end{array}$ & 2 & - & - & - & - & - & & 2 & 16 & $1-13$ \\
\hline
\end{tabular}

obligación de cuidar de otro familiar y en el $3 \%$ (n=6) por la alimentación del niño.

De acuerdo con el tiempo de estancia hospitalaria se encontró que, en el primer día, las preocupaciones más frecuentes estuvieron relacionadas con el estado emocional del niño $31.2 \%(n=5)$, en el segundo día se presentaron preocupaciones sobre el cuidado del otro hijo en el hogar, en el tercer día las preocupaciones más frecuentes fueron sobre las emociones $20 \%(n=7)$ y los servicios de salud $14.3 \%(n=5)$. En el cuarto día surgieron inquietudes sobre el entretenimiento del niño. A partir del quinto día el estado emocional, la entretención y los estudios fueron las preocupaciones persistentes.

De acuerdo con el diagnóstico, las patologías en donde se manifestaron con mayor frecuencia preocupaciones adicionales fueron las enfermedades del sistema nerviosos en el $64 \%$ $(n=7 / 19)$, psiquiátricas en el $58 \%(n=3 / 7)$ y gastrointestinales en el $52 \%(n=22 / 45)$, oncológicas $50 \%(n=1 / 2)$, respiratorias 46 $\%(n=120 / 221)$, infecciosas $36 \%(n=18 / 28)$, enfermedades de vías urinarias $40 \%(n=12 / 20)$, óseas $38 \%(n=8 / 5)$ e intervenciones quirúrgicas $32 \%(n=15 / 22)$.

El $52.4 \%(n=243)$ de los cuidadores no manifestaron preocupaciones adicionales a las médicas, esto no cambió dependiendo del tiempo de hospitalización, de la edad ni del género del niño.
Resultados relacionados con la actividad de juego seleccionada

De las 440 estrategias lúdicas realizadas, 376 actividades de juego fueron utilizadas para la estimulación general de varias habilidades y 64 fueron para el refuerzo de habilidades específicas.

Las habilidades que se estimularon a nivel general fueron en el $86.2 \%$ de los casos $(n=324)$ cognitivas, $67 \%(n=252)$ psicomotoras, $15.1 \%(n=57)$ de lenguaje o comunicación, 14.9 $\%(n=56)$ de interacción social y en el $1.3 \%(n=5)$, en el estado de ánimo.

Las habilidades específicas que se reforzaron fueron, en el $90.6 \%(n=58)$ cognitivas, en el $61 \%(n=39)$ psicomotoras, en el $20.3 \%(n=13)$ de lenguaje o comunicación, $18.7 \%(n=12)$ de interacción social y en el estado de ánimo $1.6 \%(n=1)$.

\section{Trabajo con los cuidadores}

En 25 ocasiones se involucró al cuidador de manera intencional en la actividad de juego para propiciar la interacción con el niño hospitalizado, obteniéndose todas las veces, una respuesta favorable y de participación de parte de ambos. En otras ocasiones el cuidador se involucró de manera espontánea para, facilitar el seguimiento de instrucciones en el niño, crear interés por la actividad lúdica, facilitar el desarrollo de la actividad y también como un miembro más de la actividad. 


\section{Finalización de la estrategia}

En la mayoría de los casos, de manera espontánea, los cuidadores expresaron gratitud por el desarrollo de esta estrategia lúdica y se percibió una mejora del estado de ánimo en ellos también. Cuando fue requerido por el cuidador se explicó a profundidad sobre la forma como la lúdica favorecía el proceso de hospitalización y adaptación del niño y se enseñaron actividades lúdicas específicas para el hogar.

\section{Discusión}

La vivencia de la hospitalización en el paciente pediátrico genera alteraciones contextuales y psico socioemocionales que le afectan a él y a sus cuidadores, generando necesidades que pueden pasar desapercibidas durante la atención médica, pero que se vuelven más evidentes en niños con estancias hospitalarias prolongadas o en aquellos casos en los que el curso la patología requiere de hospitalizaciones frecuentes.

Este estudio muestra que el paciente pediátrico hospitalizado presenta desde el primer día de ingreso, necesidades relacionadas con el estado de ánimo y con la interacción del cuidador, las cuales se presentaron más frecuentemente durante las primeras tres semanas de hospitalización, lo que puede estar relacionado con las estrategias de adaptación y afrontamiento empleadas por el niño para enfrentar situaciones adversas (5). Estas se presentaron independientemente de la edad del niño y su patología, aunque fueron más frecuentes en las enfermedades gastrointestinales y del sistema nervioso a comparación de las enfermedades de la piel o de las óseas. También se identificaron necesidades a nivel cognitivo y de comunicación, pero no a nivel psicomotor, lo cual es coincidente con lo reportado en estudios previos (8-10) en los que el estado de ánimo fue la principal y más frecuente afectación negativa reportada.

Las necesidades manifestadas por los cuidadores se relacionaron durante los primeros días principalmente con la incertidumbre del diagnóstico y pronóstico, dificultad de adaptación personal y familiar a las nuevas rutinas, lo cual se considera como algo esperable dentro del proceso de ajuste que supone este cambio en sus vidas (18). Muchos de ellos, contaron con apoyo de otras personas como sus padres, hermanos y pareja, que adoptaron el rol de cuidador, sin embargo, en muchos otros casos los cuidadores no contaron con red de apoyo.

Otras preocupaciones reportadas por ellos, después de la primera semana de hospitalización se relacionaron con el servicio recibido y la falta o necesidad de soporte emocional, recreativo y motivacional dentro del hospital. Estas valoraciones permiten dar cuenta, de ese momento en el que la crisis inicial de los cuidadores ha sido superada, y se comienzan a evidenciar otros aspectos importantes de vida que se ven interrumpidos. Los hallazgos de este estudio, en relación con las preocupaciones y necesidades de los cuidadores coinciden en su mayoría con otros estudios reportados en la literatura (10).
Las limitaciones de este estudio estuvieron relacionadas con la muestra pequeña en población adolescente y en pacientes con estancia hospitalaria prolongada en comparación con edades menores y estancias cortas en donde el tamaño de la muestra es mayor y podría sesgar los resultados obtenidos hacia esas variables. Dentro de las fortalezas se encuentra el amplio espectro de patologías por las que fueron ingresados los pacientes, que permitió identificar particularidades en la presencia/ausencia de las necesidades y en la percepción tanto de niños como de sus cuidadores, que podrían permitir realizar intervenciones más personalizadas.

Por último, estudios recientes exhortan sobre la pertinencia de establecer la psicopedagogía hospitalaria como «un servicio (...) desde el que se articulen las funciones de apoyo y ayuda psicológica, educativa y social al paciente y su familia (...) adaptado a la edad y estado de salud» (19) y dentro de nuestra experiencia se concuerda en que la articulación de la atención clínica con estrategias desde la psicopedagogía que sean adaptadas a las circunstancias particulares y necesidades de cada paciente, mejora la experiencia de la hospitalización para los niños y sus familias convirtiéndolo en una vivencia mucho más positiva.

Para futuras investigaciones se considera importante evaluar de manera prospectiva el impacto de las intervenciones basadas en la lúdicas en desenlaces clínicos, así como su efecto en la mitigación de las necesidades percibidas de los pacientes pediátricos hospitalizados.

\section{Conclusiones}

Los pacientes pediátricos hospitalizados independientemente de su edad o patología de ingreso, presentan durante las tres primeras semanas de hospitalización necesidades relacionadas con el estado de ánimo y la interacción con el cuidador, que pasan desapercibidas durante la atención médica; Los cuidadores presentan necesidades relacionadas con el manejo de la incertidumbre del diagnóstico y pronóstico del niño, el manejo de la interrupción en la dinámica y funcionamiento familiar, y preocupaciones con la falta de ayudas médicas, educativas y psicosociales para el niño durante su estancia en el hospital.

En nuestra experiencia, las estrategias pedagógicas basadas en la lúdica son un recurso valioso con el que las instituciones prestadoras de servicios podrían mejorar la experiencia de los niños que requieren ser hospitalizados y de sus cuidadores, a partir de la identificación e intervención de esas necesidades que pasan desapercibidas durante la atención médica, pero que afectan la vivencia y afrontamiento de una hospitalización.

\section{Agradecimientos}

Los autores desean agradecer a Lina Paola Medina, Jessica Alejandra Díaz, María Fernanda Vargas, Karen Martínez y Cindy Lorena Escamilla, estudiantes de la Universidad La Sabana y a Susan Ortigoza Torres, Adriana Bustos, Sofía Ramos Bunch, Ruth 
Alejandra Beltrán, María Fernanda Restrepo, Paula Andrea García, Alejandra Quintero y Stephanie Correal, de la Universidad El Bosque, por su participación en la implementación de la estrategia lúdica bajo la modalidad de profesional en formación.

Conflictos de interés: Ninguno.

Financiación: Ninguno.

R E F E R E N C I A S

1. López M.N, Álvarez-Llanes E. Aspectos psicológicos de la hospitalización infantil. Bol Pediatr 1996; 36: 235-240.

2. Wilson M., Megel M., Enenbach L., Carlson K. The Voices of Children: Stories About of Hospitalization; J Pediatr Health Care. 2010; 24(2): 95-102.

3. López I., Fernández A. Alteraciones emocionales en la hospitalización infantil. Tesis de grado de doctorado. Universidad de Granada, España. [Internet] 2011. [Consultado el 05 de febrero de 2020] Disponible en: https://hera.ugr.es/tesisugr/20314528. pdf

4. Villalba M. Factores psicosociales asociados a la ansiedad en niños y adolescentes hospitalizados en la fundación HOMI. Tesis de grado. Fundación Universitaria Los Libertadores, Colombia. [Internet] 2016. [Consultado el 01 de febrero de 2020] Disponible en: https://repository.libertadores.edu.co/bitstream/ handle/11371/766/VillalbaUgarteMar\%C3\%ACaYanira. pdf? sequence $=2$.

5. Delvecchio E, Salcuni S, Lis A, Germani A, Di Riso D. Hospitalized Children: Anxiety, Coping Strategies, and Pretend Play. Front Public Health. 2019; 7: 250.

6. National Center for Health Statistics. Health, United States 2018. [Internet]. 2018 [consultado el 06 de febrero de 2020]. Disponible en: https://www.cdc.gov/nchs/data/hus/2018/039.pdf

7. Asociación colombiana de empresas de medicina integral. Cifras e indicadores del Sistema de Salud [Internet] 2013. [Consultado el 30 de noviembre de 2020]. Disponible en: https://www. minsalud.gov.co/sites/rid/Lists/BibliotecaDigital/RIDE/INEC/ salude_en_cifras-2013.pdf

8. Foster K., Young A., Mitchell R., Van C., Curtis K. Experiences and needs of parents of critically injured children during the acute hospital phase: A qualitative investigation. Injury, 2017; 48(1): 114-120.

9. Rennick JE, Dougherty G, Chambers C, Stremler R, Childerhose JE, Stack DM, Harrison D, Campbell-Yeo M, Dryden-Palmer K, Zhang X, Hutchison J. Children's psychological and behavioral responses following pediatric intensive care unit hospitalization: the caring intensively study. BMC Pediatr. 2014; 14: 276.

10. Abela KM, Wardell D, Rozmus C, LoBiondo-Wood G. Impact of Pediatric Critical Illness and Injury on Families: An Updated Systematic Review. J Pediatr Nurs. 2020; 51: 21-31.

11. Ullan AM, Belver MH. Play as a Source of Psychological WellBeing for Hospitalized Children: Study Review. Int Ped Chi Care. 2019; 2(1): 92-98.

12. Koukourikos K, Tzeha L, Pantelidou P, Tsaloglidou A. The importance of play during hospitalization of children. Mater Sociomed. 2015; 27(6): 438-441.

13. Everhart JL, Haskell H, Khan A. Patient- and Family-Centered Care: Leveraging Best Practices to Improve the Care of Hospitalized Children. Pediatr Clin North Am. 2019; 66(4): 775789.
14. Hamui-Sutton A. Un acercamiento a los métodos mixtos de investigación en educación médica. Investigación en Educación Médica. 2013; 2(8): 211-216.

15. Vigotsky L. El desarrollo de los procesos psicológicos superiores. Primera edición, Barcelona, Editorial CRITICA, Grupo editorial Grijalbo, 1978.

16. Linares A. Desarrollo cognitivo: Las teorías de Piaget y de Vygotsky. Universidad Autónoma de Barcelona. [Internet] 2009 [consultado el 05 de febrero de 2020]. Disponible en: http:// www.paidopsiquiatria.cat/files/teorias_desarrollo_cognitivo. pdf

17. Ministerio de Educación Nacional de Colombia. Bases curriculares para la Educación inicial y prescolar. [Internet] 2017. [Consultado el 06 de marzo de 2020]. Disponible en: https://www.mineducacion.gov.co/1759/articles-341880_ recurso_1.pdf

18. Hasan Tehrani T, Haghighi M, Bazmamoun H. Effects of stress on mothers of hospitalized children in a hospital in iran. Iran J Child Neurol. 2012; 6(4): 39-45.

19. Garcia Docampo L. La acción psicopedagógica en el marco de la Pedagogía Hospitalaria. Tesis de maestría. Universidad de Barcelona. [Internet] 2017 [consultado el 26 de noviembre de 2020] Disponible en: http://diposit.ub.edu/dspace/ bitstream/2445/117206/1/TFM_Laura_Garcia_Docampo.pdf 